Volume 359, Number 12, December 2007, Pages 5761-5776

S 0002-9947(07)04272-9

Article electronically published on July 3, 2007

\title{
A SHARP FORM OF THE MOSER-TRUDINGER INEQUALITY ON A COMPACT RIEMANNIAN SURFACE
}

\author{
YUNYAN YANG
}

\begin{abstract}
In this paper, a sharp form of the Moser-Trudinger inequality is established on a compact Riemannian surface via the method of blow-up analysis, and the existence of an extremal function for such an inequality is proved.
\end{abstract}

\section{INTRODUCTION AND MAIN RESULTS}

Let $\Omega \subset \mathbb{R}^{2}$ be a smooth bounded domain and $H_{0}^{1}(\Omega)$ be the Sobolev space consisting of functions which vanish on the boundary of $\Omega$ and whose gradient is in $L^{2}(\Omega)$. The famous Moser-Trudinger inequality (see Moser [14]; Trudinger [17]) states the following:

$$
\sup _{u \in H_{0}^{1}(\Omega),\|\nabla u\|_{2}=1} \int_{\Omega} e^{4 \pi u^{2}} d x<+\infty .
$$

For any $p>4 \pi$, there exists a sequence $\left\{u_{\epsilon}\right\}_{\epsilon>0} \subset H_{0}^{1}(\Omega)$ with $\left\|\nabla u_{\epsilon}\right\|_{2}=1$ verifying that $\int_{\Omega} e^{p u_{\epsilon}^{2}} d x \rightarrow+\infty$. On the other hand, $\forall u \in H_{0}^{1}(\Omega), \int_{\Omega} e^{p u^{2}} d x<+\infty$ for any $p>0$. Furthermore, P. L. Lions [13] obtained the following:

Theorem A (Lions). Let $\left\{u_{\epsilon}\right\}_{\epsilon>0} \subset H_{0}^{1}(\Omega)$ with $\left\|\nabla u_{\epsilon}\right\|_{2}=1$ such that $u_{\epsilon} \rightarrow u_{0}$ weakly in $H_{0}^{1}(\Omega)$. Then for any $p<1 /\left(1-\left\|\nabla u_{0}\right\|_{2}^{2}\right)$,

$$
\limsup _{\epsilon \rightarrow 0} \int_{\Omega} e^{4 \pi p u_{\epsilon}^{2}} d x<+\infty .
$$

When $u_{\epsilon} \rightarrow u_{0}$ weakly in $H_{0}^{1}(\Omega)$ and $u_{0} \neq 0,(1.2)$ gives more precise information than (1.1). But if $u_{0}=0,(1.2)$ is a consequence of (1.1). However Adimurthi and Druet [1] proved the following:

Theorem B (Adimurthi-Druet). Let $\Omega$ be a smooth bounded domain in $\mathbb{R}^{2}$ and let $\lambda_{1}(\Omega)>0$ be the first eigenvalue of the Laplacian with Dirichlet boundary condition in $\Omega$. Then we have (i) for any $0 \leq \alpha<\lambda_{1}(\Omega)$,

$$
\sup _{u \in H_{0}^{1}(\Omega),\|\nabla u\|_{2}=1} \int_{\Omega} e^{4 \pi u^{2}\left(1+\alpha\|u\|_{2}^{2}\right)} d x<+\infty,
$$

and (ii) for any $\alpha \geq \lambda_{1}(\Omega)$,

$$
\sup _{u \in H_{0}^{1}(\Omega),\|\nabla u\|_{2}=1} \int_{\Omega} e^{4 \pi u^{2}\left(1+\alpha\|u\|_{2}^{2}\right)} d x=+\infty .
$$

Received by the editors June 7, 2005.

2000 Mathematics Subject Classification. Primary 58J05; Secondary 46E35.

Key words and phrases. Moser-Trudinger inequality, blow-up analysis, extremal function. 
This result is of a different nature from Theorem A. When $u_{\epsilon} \rightarrow u_{0}$ weakly in $H_{0}^{1}(\Omega)$ and $u_{0} \neq 0$, the inequality in Theorem $\mathrm{B}$ is a consequence of Theorem $\mathrm{A}$. But Theorem B gives new information when $u_{\epsilon} \rightarrow 0$ weakly in $H_{0}^{1}(\Omega)$.

In this paper, we consider the same inequalities as that of Theorem B on a compact Riemannian surface. Let $(\Sigma, g)$ be a compact Riemannian surface without boundary, $H^{1,2}(\Sigma)$ the completion of $C^{\infty}(\Sigma)$ in the norm

$$
\|u\|_{H^{1,2}(\Sigma)}^{2}=\int_{\Sigma}\left(|u|^{2}+|\nabla u|^{2}\right) d V_{g}<+\infty .
$$

Denote

$$
\mathcal{H}=\left\{u \in H^{1,2}(\Sigma):\|\nabla u\|_{2}=1, \int_{\Sigma} u d V_{g}=0\right\}
$$

here and in the sequel, $\|\cdot\|_{p}$ denotes the $L^{p}$-norm $\left(\int_{\Sigma}|\cdot|{ }^{p} d V_{g}\right)^{1 / p}$. Recall that the first eigenvalue of the Laplacian on $\Sigma$ is defined by

$$
\lambda_{1}(\Sigma)=\inf _{u \in H^{1,2}(\Sigma), \int_{\Sigma} u d V_{g}=0, u \neq 0} \frac{\|\nabla u\|_{2}^{2}}{\|u\|_{2}^{2}} .
$$

Write

$$
C_{\alpha}(\Sigma)=\sup _{u \in \mathcal{H}} \int_{\Sigma} e^{4 \pi u^{2}\left(1+\alpha\|u\|_{2}^{2}\right)} d V_{g}
$$

For simplicity, we denote

$$
J_{\beta}^{\alpha}(u)=\int_{\Sigma} e^{\beta u^{2}\left(1+\alpha\|u\|_{2}^{2}\right)} d V_{g}
$$

Then we can state our main results as follows:

Theorem 1.1. Let $(\Sigma, g)$ be a compact Riemannian surface without boundary. Then we have

(1) For any $0 \leq \alpha<\lambda_{1}(\Sigma), C_{\alpha}(\Sigma)<+\infty$.

(2) For any $\alpha \geq \lambda_{1}(\Sigma), C_{\alpha}=+\infty$.

(3) For sufficiently small $\alpha>0$, there exists a $u_{\alpha} \in \mathcal{H}$ such that $C_{\alpha}(\Sigma)=$ $J_{4 \pi}^{\alpha}\left(u_{\alpha}\right)$.

As we have explained before, this result gives more information than the usual Moser-Trudinger inequality on a compact Riemannian surface (see for example [9], [10]). We follow the lines of the proof of Adimurthi and Druet [1]. First, we choose test functions to prove (2). Then we use blow-up analysis to prove (1), and finally use the capacity technique to prove (3).

Similarly, we have the following:

Theorem 1.2. Let $(\Sigma, g)$ be a compact Riemannian surface without boundary. Denote

$$
\mathcal{H}_{1}=\left\{u \in H^{1,2}(\Sigma): \int_{\Sigma}\left(|u|^{2}+|\nabla u|^{2}\right) d V_{g}=1\right\}
$$

and

$$
C_{\alpha}^{1}(\Sigma)=\sup _{u \in \mathcal{H}_{1}} \int_{\Sigma} e^{4 \pi u^{2}\left(1+\alpha\|u\|_{2}^{2}\right)} d V_{g}
$$

Then we have

(1) For any $0 \leq \alpha<\lambda_{1}(\Sigma), C_{\alpha}^{1}(\Sigma)<+\infty$.

(2) For any $\alpha \geq \lambda_{1}(\Sigma), C_{\alpha}^{1}(\Sigma)=+\infty$. 
(3) For sufficiently small $\alpha>0$, there exists a $u_{\alpha} \in \mathcal{H}_{1}$ such that $C_{\alpha}^{1}(\Sigma)=$ $J_{4 \pi}^{\alpha}\left(u_{\alpha}\right)$.

Since the proof of Theorem 1.2 is completely analogous to that of Theorem 1.1, we omit it in this paper.

Extremal functions for critical functionals can be obtained by the method of blow-up analysis. In 1984, Schoen [15] solved the Yamabe problem. In 1986, Escobar and Schoen [7] found conformal metrics with prescribed curvatures in high dimensions. In 1997, Ding, Jost, Li and Wang [5] proved the solvability of the equation $\triangle u=8 \pi-h e^{8 \pi u}$ on a compact Riemannian surface. For the existence of extremal functions for the classical Moser-Trudinger inequality, we would like to mention Carleson and Chang [3], Flucher [8], Lin [12], Li [10] and Li-Liu [11]. About extremals for optimal Sobolev inequalities on Riemannian manifolds, we refer the reader to Druet and Hebey [6] and the references therein.

Throughout this paper we denote the Laplacian and the gradient on $\Sigma$ by $\triangle$ and $\nabla$, those on $\mathbb{R}^{2}$ by $\triangle_{\mathbb{R}^{2}}$ and $\nabla_{\mathbb{R}^{2}}$ respectively.

We organize this paper as follows: In section 2 , we construct test functions to prove point (2) of Theorem 1.1. In section 3, we prove the existence of a maximizer of a subcritical functional $J_{4 \pi-\epsilon}^{\alpha}$, and give the corresponding Euler-Lagrange equation. Section 4 contributes to the asymptotic behavior of the maximizers through blow-up analysis. An upper bound of $J_{4 \pi}^{\alpha}$ is derived in section 5 under the assumption that blow-up occurs. In the last section, we construct a sequence of functions to show that the upper bound of $J_{4 \pi}^{\alpha}$ is in fact greater than the one we derived in section 5 .

\section{The TEST FUnCTIONS}

In this section, following Adimurthi and Druet [1], we choose test functions to prove point (2) of Theorem 1.1. Let $u_{0}$ be a weak solution of

$$
\left\{\begin{array}{l}
-\triangle u_{0}=\lambda_{1}(\Sigma) u_{0} \quad \text { in } \quad \Sigma \\
\int_{\Sigma} u_{0} d V_{g}=0, \quad\left\|u_{0}\right\|_{2}^{2}=1 .
\end{array}\right.
$$

By elliptic estimates, $u_{0} \in C^{\infty}(\Sigma)$. The fact that $\int_{\Sigma} u_{0} d V_{g}=0$ implies that there exists some $p \in \Sigma$ such that $u_{0}(p)>0$, and a domain $U \subset \Sigma$ such that $p \in U$ and $u_{0} \geq u_{0}(p) / 2$ in $U$. Choose an isothermal coordinate system $(V, \psi)$ around $p$ such that $V \subset U, \psi: V \rightarrow \mathbb{B}_{\delta}=\left\{x \in \mathbb{R}^{2}:|x| \leq \delta\right\}, \psi(p)=0$. In this coordinate system, the metric $g$ can be represented by $g=e^{2} f\left(d x_{1}^{2}+d x_{2}^{2}\right)$, where $f$ is a smooth function with $f(0)=0$.

For any $x \in \mathbb{B}_{\delta}$, let

$$
m_{\epsilon}(x)=\left\{\begin{array}{l}
\sqrt{\frac{1}{4 \pi} \log \frac{1}{\epsilon}}, \quad|x| \leq \delta \sqrt{\epsilon}, \\
\frac{1}{\sqrt{\pi \log \frac{1}{\epsilon}}} \log \frac{\delta}{|x|}, \quad \delta \sqrt{\epsilon}<|x| \leq \delta .
\end{array}\right.
$$

We set

$$
u_{\epsilon}=\left\{\begin{array}{lll}
m_{\epsilon} \circ \psi & \text { in } & \psi^{-1}\left(\mathbb{B}_{\delta}\right), \\
l_{\epsilon} \varphi & \text { in } & \Sigma \backslash \psi^{-1}\left(\mathbb{B}_{\delta}\right),
\end{array}\right.
$$

where $\varphi \in C_{0}^{\infty}\left(\Sigma \backslash \psi^{-1}\left(\mathbb{B}_{\delta}\right)\right)$ and $l_{\epsilon}$ is a real number such that $\int_{\Sigma} u_{\epsilon} d V_{g}=0$. 
It is not difficult to check that

$$
\begin{array}{cl}
l_{\epsilon}=O\left(1 /\left(\log \frac{1}{\epsilon}\right)^{1 / 2}\right), & \left\|\nabla u_{\epsilon}\right\|_{2}^{2}=1+O\left(1 / \log \frac{1}{\epsilon}\right), \\
\left\|u_{\epsilon}\right\|_{1}=O\left(1 /\left(\log \frac{1}{\epsilon}\right)^{1 / 2}\right), & \left\|u_{\epsilon}\right\|_{2}^{2}=O\left(1 / \log \frac{1}{\epsilon}\right) .
\end{array}
$$

Setting $v_{\epsilon}=u_{\epsilon}+t_{\epsilon} u_{0}$ with $t_{\epsilon} \rightarrow 0$, we get $t_{\epsilon}^{2} \log \frac{1}{\epsilon} \rightarrow+\infty$ and $t_{\epsilon}^{2}\left(\log \frac{1}{\epsilon}\right)^{1 / 2} \rightarrow 0$. Then we have

$$
\begin{gathered}
\left\|v_{\epsilon}\right\|_{2}^{2}=\left\|u_{\epsilon}\right\|_{2}^{2}+t_{\epsilon}^{2}\left\|u_{0}\right\|_{2}^{2}+2 t_{\epsilon} \int_{\Sigma} u_{\epsilon} u_{0} d V_{g} \\
=t_{\epsilon}^{2}+2 t_{\epsilon} \int_{\Sigma} u_{\epsilon} u_{0} d V_{g}+O\left(1 / \log \frac{1}{\epsilon}\right) \\
\left\|\nabla v_{\epsilon}\right\|_{2}^{2}=\left\|\nabla u_{\epsilon}\right\|_{2}^{2}+t_{\epsilon}^{2}\left\|\nabla u_{0}\right\|_{2}^{2}+2 t_{\epsilon} \int_{\Sigma} \nabla u_{\epsilon} \nabla u_{0} d V_{g} \\
=1+2 \lambda_{1}(\Sigma) t_{\epsilon} \int_{\Sigma} u_{\epsilon} u_{0} d V_{g}+\lambda_{1}(\Sigma) t_{\epsilon}^{2}+O\left(1 / \log \frac{1}{\epsilon}\right), \\
\frac{1}{\left\|\nabla v_{\epsilon}\right\|_{2}^{2}}\left(1+\alpha \frac{\left\|v_{\epsilon}\right\|_{2}^{2}}{\left\|\nabla v_{\epsilon}\right\|_{2}^{2}}\right)=1+\left(\alpha-\lambda_{1}(\Sigma)\right)\left(t_{\epsilon}^{2}+2 t_{\epsilon} \int_{\Sigma} u_{\epsilon} u_{0} d V_{g}\right) \\
+o\left(t_{\epsilon} /\left(\log \frac{1}{\epsilon}\right)^{1 / 2}\right) .
\end{gathered}
$$

We have for $\alpha \geq \lambda_{1}(\Sigma)$,

$$
\frac{1}{\left\|\nabla v_{\epsilon}\right\|_{2}^{2}}\left(1+\alpha \frac{\left\|v_{\epsilon}\right\|_{2}^{2}}{\left\|\nabla v_{\epsilon}\right\|_{2}^{2}}\right) \geq 1+o\left(t_{\epsilon} /\left(\log \frac{1}{\epsilon}\right)^{1 / 2}\right) .
$$

Note that on $\psi^{-1}\left(\mathbb{B}_{\delta \sqrt{\epsilon}}\right)$,

$$
\begin{aligned}
4 \pi \frac{v_{\epsilon}^{2}}{\left\|\nabla v_{\epsilon}\right\|_{2}^{2}}\left(1+\alpha \frac{\left\|v_{\epsilon}\right\|_{2}^{2}}{\left\|\nabla v_{\epsilon}\right\|_{2}^{2}}\right) \geq & 4 \pi\left(t_{\epsilon}^{2} u_{0}^{2}+\frac{1}{4 \pi} \log \frac{1}{\epsilon}+2 t_{\epsilon}\left(\frac{1}{4 \pi} \log \frac{1}{\epsilon}\right)^{1 / 2} u_{0}\right) \\
& \times\left(1+o\left(t_{\epsilon} /\left(\log \frac{1}{\epsilon}\right)^{1 / 2}\right)\right) \\
\geq & \log \frac{1}{\epsilon}+t_{\epsilon}\left(\log \frac{1}{\epsilon}\right)^{1 / 2}\left(4 \sqrt{\pi} u_{0}+o(1)\right) .
\end{aligned}
$$

Hence

$$
\begin{aligned}
\int_{\Sigma} e^{4 \pi \frac{v_{\epsilon}^{2}}{\left\|\nabla v_{\epsilon}\right\|_{2}^{2}}\left(1+\alpha \frac{\left\|v_{\epsilon}\right\|_{2}^{2}}{\| \nabla v_{\epsilon}^{2}}\right)} d V_{g} & \geq \int_{\psi^{-1}\left(\mathbb{B}_{\delta \sqrt{\epsilon}}\right)} \frac{1}{\epsilon} e^{t_{\epsilon} \sqrt{\log \frac{1}{\epsilon}}\left(4 \sqrt{\pi} u_{0}+o(1)\right)} d V_{g} \\
& \geq C(\delta) e^{t_{\epsilon} \sqrt{\log \frac{1}{\epsilon}}\left(2 \sqrt{\pi} u_{0}(p)+o(1)\right)} .
\end{aligned}
$$

Since $u_{0}(p)>0$, then $\int_{\Sigma} e^{4 \pi \frac{v_{\epsilon}^{2}}{\left\|\nabla v_{\epsilon}\right\|_{2}^{2}}\left(1+\alpha \frac{\left\|v_{\epsilon}\right\|_{2}^{2}}{\left\|\nabla v_{\epsilon}\right\|_{2}^{2}}\right)} d V_{g} \rightarrow+\infty$ as $\epsilon \rightarrow 0$. This completes the proof of (2) of Theorem 1.1.

\section{EXISTENCE OF MAXIMIZERS FOR SUBCRITICAL FUNCTIONALS}

In this section, we will prove the existence of maximizers of subcritical functionals. Similar to P. L. Lions [13], we have the following:

Lemma 3.1. Let $\left\{u_{\epsilon}\right\}_{\epsilon>0}$ be a sequence of functions in $\mathcal{H}$ with $u_{\epsilon} \rightarrow u_{0}$ weakly in $H^{1,2}(\Sigma)$. Then for any $p<1 /\left(1-\left\|\nabla u_{0}\right\|_{2}^{2}\right)$, $\limsup _{\epsilon \rightarrow 0} \int_{\Sigma} e^{4 \pi p u_{\epsilon}^{2}} d V_{g}<+\infty$. 
Proof. Clearly we have $\int_{\Sigma} u_{0} d V_{g}=0$. If $u_{0} \neq 0$, then one can see that

$$
\left\|\nabla\left(u_{\epsilon}-u_{0}\right)\right\|_{2}^{2} \rightarrow 1-\left\|\nabla u_{0}\right\|_{2}^{2}<1 .
$$

Hence we have for $p<1 /\left(1-\left\|\nabla u_{0}\right\|_{2}^{2}\right)$,

$$
\begin{aligned}
\int_{\Sigma} e^{4 \pi p u_{\epsilon}^{2}} d V_{g} & \leq \int_{\Sigma} e^{4 \pi p(1+\delta)\left(u_{\epsilon}-u_{0}\right)^{2}+4 \pi p(1+1 / \delta) u_{0}^{2}} d V_{g} \\
& \leq\left(\int_{\Sigma} e^{4 \pi \frac{\left(u_{\epsilon}-u_{0}\right)^{2}}{\left\|\nabla\left(u_{\epsilon}-u_{0}\right)\right\|_{2}^{2}}} d V_{g}\right)^{1 / r}\left(\int_{\Sigma} e^{4 \pi p^{\prime} u_{0}^{2}} d V_{g}\right)^{1 / s}
\end{aligned}
$$

for some $\delta>0$ and $p^{\prime}>p$ provided that $\epsilon$ is sufficiently small, where $1 / r+1 / s=1$. By the Orlicz embedding, $e^{u_{0}^{2}}$ is bounded in $L^{q}(\Sigma)$ for any $q>1$. A result of Fontana [9] gives $\sup _{u \in \mathcal{H}} \int_{\Sigma} e^{4 \pi u^{2}} d V_{g}<+\infty$. Hence

$$
\limsup _{\epsilon \rightarrow 0} \int_{\Sigma} e^{4 \pi p u_{\epsilon}^{2}} d V_{g}<+\infty
$$

If $u_{0}=0,(3.1)$ is an immediate corollary of Fontana's result.

Lemma 3.2. Let $0 \leq \alpha<\lambda_{1}(\Sigma)$. For any $\epsilon>0$, there exists a $u_{\epsilon} \in C^{\infty}(\Sigma) \cap \mathcal{H}$ such that

$$
J_{4 \pi-\epsilon}^{\alpha}\left(u_{\epsilon}\right)=\sup _{u \in \mathcal{H}} J_{4 \pi-\epsilon}^{\alpha}(u)
$$

Proof. For any fixed $\epsilon>0$, we choose a maximizing sequence $\left\{u_{i}\right\} \subset \mathcal{H}$ such that

$$
J_{4 \pi-\epsilon}^{\alpha}\left(u_{i}\right) \rightarrow \sup _{u \in \mathcal{H}} J_{4 \pi-\epsilon}^{\alpha}(u) \quad \text { as } \quad i \rightarrow+\infty .
$$

Since $\left\{u_{i}\right\}$ is bounded in $H^{1,2}(\Sigma)$, we have

$$
\begin{array}{lll}
u_{i} \rightarrow u_{\epsilon} & \text { weakly in } H^{1,2}(\Sigma), \\
u_{i} \rightarrow u_{\epsilon} & \text { strongly in } L^{2}(\Sigma), \\
u_{i} \rightarrow u_{\epsilon} & \text { a.e. in } \Sigma .
\end{array}
$$

Hence

$$
f_{i}=e^{(4 \pi-\epsilon) u_{i}^{2}\left(1+\alpha\left\|u_{i}\right\|_{2}^{2}\right)} \rightarrow f_{\epsilon}=e^{(4 \pi-\epsilon) u_{\epsilon}^{2}\left(1+\alpha\left\|u_{\epsilon}\right\|_{2}^{2}\right)} \quad \text { a.e. } \quad \text { in } \quad \Sigma .
$$

If we suppose $u_{\epsilon}=0$, then we have $1+\alpha\left\|u_{i}\right\|_{2}^{2} \rightarrow 1$. Since $\int_{\Sigma} e^{4 \pi u_{i}^{2}} d V_{g}<+\infty$, we have $f_{i}$ is bounded in $L^{p}(\Sigma)$ for some $p>1$ and $f_{i} \rightarrow 1$ in $L^{1}(\Sigma)$. Hence $\operatorname{Vol}(\Sigma)=\sup _{u \in \mathcal{H}} J_{4 \pi-\epsilon}^{\alpha}(u)$, which is impossible. Therefore $u_{\epsilon} \neq 0$. By Lemma 3.1, we have for $p<1 /\left(1-\left\|\nabla u_{\epsilon}\right\|_{2}^{2}\right)$,

$$
\limsup _{i \rightarrow+\infty} \int_{\Sigma} e^{4 \pi p u_{i}^{2}} d V_{g}<+\infty
$$

Since $0 \leq \alpha<\lambda_{1}(\Sigma)$, we have

$$
1+\alpha\left\|u_{i}\right\|_{2}^{2} \rightarrow 1+\alpha\left\|u_{\epsilon}\right\|_{2}^{2}<\frac{1}{1-\left\|\nabla u_{\epsilon}\right\|_{2}^{2}}
$$

Hence $f_{i}$ is bounded in $L^{p}(\Sigma)$ for some $p>1$. Since $f_{i} \rightarrow f_{\epsilon}$ a.e. in $\Sigma$, then $f_{i} \rightarrow f_{\epsilon}$ strongly in $L^{1}(\Sigma)$. Therefore $\int_{\Sigma} f_{\epsilon} d V_{g}=\sup _{u \in \mathcal{H}} J_{4 \pi-\epsilon}^{\alpha}(u)$, and $u_{\epsilon} \in \mathcal{H}$. 
It is not difficult to check that $u_{\epsilon}$ satisfies

$$
\left\{\begin{array}{l}
-\triangle u_{\epsilon}=\frac{\beta_{\epsilon}}{\lambda_{\epsilon}} u_{\epsilon} e^{\alpha_{\epsilon} u_{\epsilon}^{2}}+\gamma_{\epsilon} u_{\epsilon}-\frac{\mu_{\epsilon}}{\lambda_{\epsilon}} \\
\int_{\Sigma} u_{\epsilon} d V_{g}=0, \quad\left\|\nabla u_{\epsilon}\right\|_{2}=1 \\
\alpha_{\epsilon}=(4 \pi-\epsilon)\left(1+\alpha\left\|u_{\epsilon}\right\|_{2}^{2}\right) \\
\beta_{\epsilon}=\left(1+\alpha\left\|u_{\epsilon}\right\|_{2}^{2}\right) /\left(1+2 \alpha\left\|u_{\epsilon}\right\|_{2}^{2}\right) \\
\gamma_{\epsilon}=\alpha /\left(1+2 \alpha\left\|u_{\epsilon}\right\|_{2}^{2}\right) \\
\lambda_{\epsilon}=\int_{\Sigma} u_{\epsilon}^{2} e^{\alpha_{\epsilon} u_{\epsilon}^{2}} d V_{g} \\
\mu_{\epsilon}=\beta_{\epsilon} \int_{\Sigma} u_{\epsilon} e^{\alpha_{\epsilon} u_{\epsilon}^{2}} d V_{g} .
\end{array}\right.
$$

\section{BLOW-UP ANALYSIS}

In this section, we will use blow-up analysis to understand the asymptotic behavior of the maximizers $u_{\epsilon}$. We proceed as Adimurthi and Druet did in [1]. Firstly we have

Lemma 4.1. $\liminf _{\epsilon \rightarrow 0} \lambda_{\epsilon}>0$.

Proof. Using the elementary inequality $e^{t} \leq 1+t e^{t}$ for $t \geq 0$, one has

$$
\int_{\Sigma} e^{\alpha_{\epsilon} u_{\epsilon}^{2}} d V_{g} \leq \operatorname{Vol}(\Sigma)+\alpha_{\epsilon} \lambda_{\epsilon}
$$

On the other hand,

$$
\lim _{\epsilon \rightarrow 0} \int_{\Sigma} e^{\alpha_{\epsilon} u_{\epsilon}^{2}} d V_{g}=\sup _{u \in \mathcal{H}} \int_{\Sigma} e^{4 \pi u^{2}\left(1+\alpha\|u\|_{2}^{2}\right)} d V_{g}>\operatorname{Vol}(\Sigma) .
$$

The above two inequalities, together with the fact that $\alpha_{\epsilon}$ is bounded, imply the result.

Lemma 4.2. $\mu_{\epsilon} / \lambda_{\epsilon}$ is bounded.

Proof. By (3.2), we have

$$
\begin{aligned}
\frac{\left|\mu_{\epsilon}\right|}{\lambda_{\epsilon}} & \leq \beta_{\epsilon} \int_{\left|u_{\epsilon}\right| \geq 1} \frac{\left|u_{\epsilon}\right|}{\lambda_{\epsilon}} e^{\alpha_{\epsilon} u_{\epsilon}^{2}} d V_{g}+\beta_{\epsilon} \int_{\left|u_{\epsilon}\right|<1} \frac{\left|u_{\epsilon}\right|}{\lambda_{\epsilon}} e^{\alpha_{\epsilon} u_{\epsilon}^{2}} d V_{g} \\
& \leq 1+\frac{e^{\alpha_{\epsilon}}}{\lambda_{\epsilon}} \operatorname{Vol}(\Sigma) \leq C ;
\end{aligned}
$$

here we have used Lemma 4.1 and $\beta_{\epsilon} \leq 1$.

Let $c_{\epsilon}=\left|u_{\epsilon}\left(x_{\epsilon}\right)\right|=\max _{\Sigma}\left|u_{\epsilon}\right|$. If $c_{\epsilon}$ is bounded, by the standard elliptic estimates, Theorem 1.1 holds. Without loss of generality, we may assume in the following that

$$
x_{\epsilon} \rightarrow p, \quad u_{\epsilon}\left(x_{\epsilon}\right) \rightarrow+\infty
$$

as $\epsilon \rightarrow 0$. Here and in the sequel, we do not distinguish sequence and subsequence; the reader can understand it easily from the context.

Lemma 4.3. $u_{\epsilon} \rightarrow 0$ weakly in $H^{1,2}(\Sigma), u_{\epsilon} \rightarrow 0$ strongly in $L^{2}(\Sigma)$, and $\left|\nabla u_{\epsilon}\right|^{2} d x \rightarrow$ $\delta_{p}$ in the sense of measure, where $\delta_{p}$ is the Dirac measure at $p$. 
Proof. We may assume $u_{\epsilon} \rightarrow u_{0}$ weakly in $H^{1,2}(\Sigma)$. Obviously $\int_{\Sigma} u_{0} d V_{g}=0$. If we suppose $u_{0} \neq 0$, then we have

$$
1+\alpha\left\|u_{\epsilon}\right\|_{2}^{2} \rightarrow 1+\alpha\left\|u_{0}\right\|_{2}^{2} \leq 1+\left\|\nabla u_{0}\right\|^{2}<\frac{1}{1-\left\|\nabla u_{0}\right\|_{2}^{2}}
$$

By Lemma 3.1, one has $e^{\alpha_{\epsilon} u_{\epsilon}^{2}}$ is bounded in $L^{p}(\Sigma)$ for some $p>1$ provided that $\epsilon$ is sufficiently small. Applying the elliptic estimates to equation (3.2), one gets $c_{\epsilon}$ is bounded, and a contradiction. Hence $u_{0}=0$, whence $\alpha_{\epsilon} \rightarrow 4 \pi, \beta_{\epsilon} \rightarrow 1$ and $\gamma_{\epsilon} \rightarrow \alpha$. Assume $\left|\nabla u_{\epsilon}\right|^{2} d V_{g} \rightarrow \mu$ in the sense of measure. If $\mu \neq \delta_{p}$ for all $p \in \Sigma$, then the usual truncation and covering arguments imply that $e^{\alpha_{\epsilon} u_{\epsilon}^{2}}$ is bounded in $L^{q}(\Sigma)$ for some $q>1$. Applying elliptic estimates again to equation (3.2), we have $u_{\epsilon}$ is bounded in $L^{\infty}(\Sigma)$, which contradicts (4.2).

Take an isothermal coordinate system $(\Omega, \phi)$ near $p$ such that $\phi(p)=0$. In such coordinates, the metric $g$ has the representation $g=e^{2 f}\left(d x_{1}^{2}+d x_{2}^{2}\right)$ with $f(0)=0$. Let $r_{\epsilon}^{2}=\frac{\lambda_{\epsilon}}{\beta_{\epsilon} c_{\epsilon}^{2}} e^{-\alpha_{\epsilon} c_{\epsilon}^{2}}$ and $\Omega_{\epsilon}=\left\{x \in \mathbb{R}^{2}: x_{\epsilon}+r_{\epsilon} x \in \Omega\right\}$. Let $\psi_{\epsilon}(x)=u\left(x_{\epsilon}+r_{\epsilon} x\right) / c_{\epsilon}$ and

$$
\varphi_{\epsilon}(x)=c_{\epsilon}\left(u_{\epsilon}\left(x_{\epsilon}+r_{\epsilon} x\right)-c_{\epsilon}\right), \quad x \in \Omega_{\epsilon} .
$$

Then we have the following:

Lemma 4.4. For any $0<\alpha<4 \pi$, we have $r_{\epsilon}^{2} e^{\alpha c_{\epsilon}^{2}} \rightarrow 0$.

Proof. A straightforward calculation shows that, for $0<\alpha<4 \pi$,

$$
\begin{aligned}
r_{\epsilon}^{2} e^{\alpha c_{\epsilon}^{2}} & =\frac{e^{\left(\alpha-\alpha_{\epsilon}\right) c_{\epsilon}^{2}}}{\beta_{\epsilon} c_{\epsilon}^{2}} \int_{\Sigma} u_{\epsilon}^{2} e^{\alpha_{\epsilon} u_{\epsilon}^{2}} d V_{g} \\
& \leq \frac{1}{\beta_{\epsilon} c_{\epsilon}^{2}} \int_{\Sigma} u_{\epsilon}^{2} e^{\alpha u_{\epsilon}^{2}} d V_{g}
\end{aligned}
$$

for sufficiently small $\epsilon$. Obviously $u_{\epsilon}^{2} e^{\alpha u_{\epsilon}^{2}}$ is bounded in $L^{1}(\Sigma)$, which gives the result immediately.

By (3.2), we have

$$
\left\{\begin{array}{l}
-\triangle \psi_{\epsilon}=\frac{1}{c_{\epsilon}^{2}} \psi_{\epsilon} e^{\alpha_{\epsilon}\left(u_{\epsilon}^{2}-c_{\epsilon}^{2}\right)}+r_{\epsilon}^{2} \gamma_{\epsilon} \psi_{\epsilon}-r_{\epsilon}^{2} \frac{\mu_{\epsilon}}{c_{\epsilon} \lambda_{\epsilon}}, \\
-\triangle \varphi_{\epsilon}=\psi_{\epsilon} e^{\alpha_{\epsilon} \varphi_{\epsilon}\left(1+\psi_{\epsilon}\right)}+c_{\epsilon} r_{\epsilon}^{2} \gamma_{\epsilon} u_{\epsilon}\left(x_{\epsilon}+r_{\epsilon} x\right)-c_{\epsilon} r_{\epsilon}^{2} \mu_{\epsilon} / \lambda_{\epsilon} .
\end{array}\right.
$$

It is easy to see that $\Delta \psi_{\epsilon} \rightarrow 0$ in $L^{2}\left(B_{R}(0)\right),\left|\psi_{\epsilon}\right| \leq 1$ and $\psi_{\epsilon}(0)=1$. Elliptic estimates and Liouville's theorem give $\psi_{\epsilon} \rightarrow 1$ in $C^{2}\left(B_{R / 2}(0)\right)$. Applying elliptic estimates to (4.3), we obtain $\varphi_{\epsilon} \rightarrow \varphi$ in $C_{l o c}^{2}\left(\mathbb{R}^{2}\right)$, where $\varphi$ satisfies

$$
\left\{\begin{array}{l}
-\Delta_{\mathbb{R}^{2}} \varphi=e^{8 \pi \varphi} \text { in } \mathbb{R}^{2} \\
\varphi(0)=\sup _{\mathbb{R}^{2}} \varphi=0 \\
\int_{\mathbb{R}^{2}} e^{8 \pi \varphi} d x \leq 1
\end{array}\right.
$$

By the uniqueness result of Chen and $\operatorname{Li}[4]$, we have $\varphi(x)=-\frac{1}{4 \pi} \log \left(1+\pi|x|^{2}\right)$ and $\int_{\mathbb{R}^{2}} e^{8 \pi \varphi} d x=1$.

Define $u_{\epsilon, \beta}=\min \left\{\beta c_{\epsilon}, u_{\epsilon}\right\}$. Then we have

Lemma 4.5. $\forall 0<\beta<1, \quad \lim \sup _{\epsilon \rightarrow 0}\left\|\nabla u_{\epsilon, \beta}\right\|_{2}^{2}=\beta$. 
Proof. By the fact that $\psi_{\epsilon} \rightarrow 1$ in $C_{l o c}^{2}\left(\mathbb{R}^{2}\right)$, we have

$$
B_{R r_{\epsilon}}\left(x_{\epsilon}\right) \subset\left\{x \in \Sigma: u_{\epsilon}(x) \geq \beta c_{\epsilon}\right\}
$$

for any fixed $R>0$ and sufficiently small $\epsilon$. Using the divergence theorem and equation (3.2), we have

$$
\begin{aligned}
\int_{\Sigma}\left|\nabla\left(u_{\epsilon}-\beta c_{\epsilon}\right)^{+}\right|^{2} d V_{g} & =-\int_{\Sigma}\left(u_{\epsilon}-\beta c_{\epsilon}\right)^{+} \triangle u_{\epsilon} d V_{g} \\
& =\int_{u_{\epsilon} \geq \beta c_{\epsilon}}\left(u_{\epsilon}-\beta c_{\epsilon}\right) \frac{1}{\lambda_{\epsilon}} u_{\epsilon} e^{\alpha_{\epsilon} u_{\epsilon}^{2}} d V_{g}+o_{\epsilon}(1) \\
& \geq \int_{B_{R r_{\epsilon}}\left(x_{\epsilon}\right)}\left(u_{\epsilon}-\beta c_{\epsilon}\right) \frac{1}{\lambda_{\epsilon}} u_{\epsilon} e^{\alpha_{\epsilon} u_{\epsilon}^{2}} d V_{g}+o_{\epsilon}(1) \\
& =(1-\beta) \int_{B_{R}(0)} e^{8 \pi \varphi} d x+o_{\epsilon}(1)+o_{\epsilon}(R)
\end{aligned}
$$

where $o_{\epsilon}(1) \rightarrow 0$ as $\epsilon \rightarrow 0$, and $o_{\epsilon}(R) \rightarrow 0$ for any fixed $R$ as $\epsilon \rightarrow 0$. Letting $\epsilon \rightarrow 0$ first, then $R \rightarrow+\infty$, we obtain

$$
\liminf _{\epsilon \rightarrow 0} \int_{\Sigma}\left|\nabla\left(u_{\epsilon}-\beta c_{\epsilon}\right)^{+}\right|^{2} d V_{g} \geq 1-\beta
$$

Using the divergence theorem, equation (3.2), Lemma 4.2, Lemma 4.3 and the fact that $\varphi_{\epsilon} \rightarrow \varphi$ in $C_{l o c}^{2}\left(\mathbb{R}^{2}\right)$, we have

$$
\begin{aligned}
\int_{\Sigma}\left|\nabla u_{\epsilon, \beta}\right|^{2} d V_{g} & =-\int_{\Sigma} u_{\epsilon, \beta} \triangle u_{\epsilon} d V_{g} \\
& =\int_{\Sigma} u_{\epsilon, \beta} \frac{1}{\lambda_{\epsilon}} u_{\epsilon} e^{\alpha_{\epsilon} u_{\epsilon}^{2}} d V_{g}+o_{\epsilon}(1) \\
& \geq \int_{B_{R \epsilon}\left(x_{\epsilon}\right)} \frac{1}{\lambda_{\epsilon}} \beta c_{\epsilon} u_{\epsilon} e^{\alpha_{\epsilon} u_{\epsilon}^{2}} d V_{g}+o_{\epsilon}(1) \\
& =\beta \int_{B_{R}(0)} e^{8 \pi \varphi} d x+o_{\epsilon}(1)+o_{\epsilon}(R)
\end{aligned}
$$

Letting $\epsilon \rightarrow 0$ first, then $R \rightarrow+\infty$, we have $\liminf _{\epsilon \rightarrow 0} \int_{\Sigma}\left|\nabla u_{\epsilon, \beta}\right|^{2} d V_{g} \geq \beta$. Noting that

$$
\int_{\Sigma}\left|\nabla\left(u_{\epsilon}-\beta c_{\epsilon}\right)^{+}\right|^{2} d V_{g}+\int_{\Sigma}\left|\nabla u_{\epsilon, \beta}\right|^{2} d V_{g}=1,
$$

we get the result.

Lemma 4.6. $\lim \sup _{\epsilon \rightarrow 0} J_{4 \pi-\epsilon}^{\alpha}\left(u_{\epsilon}\right) \leq \operatorname{Vol}(\Sigma)+\lim \sup _{\epsilon \rightarrow 0} \frac{\lambda_{\epsilon}}{c_{\epsilon}^{2}}$.

Proof. For any $0<\beta<1$, an elementary computation gives

$$
\begin{aligned}
J_{4 \pi-\epsilon}^{\alpha}\left(u_{\epsilon}\right) & =\int_{u_{\epsilon}<\beta c_{\epsilon}} e^{\alpha_{\epsilon} u_{\epsilon}^{2}} d V_{g}+\int_{u_{\epsilon} \geq \beta c_{\epsilon}} e^{\left(\alpha_{\epsilon}\right) u_{\epsilon}^{2}} d V_{g} \\
& \leq \int_{\Sigma} e^{\alpha_{\epsilon} u_{\epsilon, \beta}^{2}} d V_{g}+\frac{\lambda_{\epsilon}}{\beta^{2} c_{\epsilon}^{2}}
\end{aligned}
$$


By Lemma 4.5, we can see that $e^{\alpha_{\epsilon} u_{\epsilon, \beta}^{2}}$ is bounded in $L^{p}(\Sigma)$ for some $p>1$, whence $\int_{\Sigma} e^{\alpha_{\epsilon} u_{\epsilon, \beta}^{2}} d V_{g} \rightarrow \operatorname{Vol}(\Sigma)$ as $\epsilon \rightarrow 0$. Therefore

$$
J_{4 \pi-\epsilon}^{\alpha}\left(u_{\epsilon}\right) \leq \operatorname{Vol}(\Sigma)+\frac{\lambda_{\epsilon}}{\beta^{2} c_{\epsilon}^{2}}+o_{\epsilon}(1),
$$

where $o_{\epsilon}(1) \rightarrow 0$ as $\epsilon \rightarrow 0$. Letting $\epsilon \rightarrow 0$ first, then $\beta \rightarrow 1$, we obtain the result.

Similar to [1] and [10], we have the following:

Lemma 4.7. For any $\phi \in C^{\infty}(\Sigma)$ we have

$$
\lim _{\epsilon \rightarrow 0} \int_{\Sigma} \phi \frac{\beta_{\epsilon}}{\lambda_{\epsilon}} c_{\epsilon} u_{\epsilon} e^{\alpha_{\epsilon} u_{\epsilon}^{2}} d x=\phi(p) .
$$

Proof. We divide $\Sigma$ into three parts:

$$
\Sigma=\left(\left\{u_{\epsilon}>\beta c_{\epsilon}\right\} \backslash B_{R r_{\epsilon}}\left(x_{\epsilon}\right)\right) \cup\left(\left\{u_{\epsilon} \leq \beta c_{\epsilon}\right\} \backslash B_{R r_{\epsilon}}\left(x_{\epsilon}\right)\right) \cup B_{R r_{\epsilon}}\left(x_{\epsilon}\right),
$$

for some $0<\beta<1$. Denote the integrals on the left side of (4.5) on the above three domains by $I_{1}, I_{2}$ and $I_{3}$ respectively. Then

$$
\begin{aligned}
\left|I_{1}\right| & \leq \frac{1}{\beta} \sup _{\Sigma}|\phi| \int_{\left\{u_{\epsilon}>\beta c_{\epsilon}\right\} \backslash B_{R r_{\epsilon}}\left(x_{\epsilon}\right)} \frac{1}{\lambda_{\epsilon}} u_{\epsilon}^{2} e^{\alpha_{\epsilon} u_{\epsilon}^{2}} d V_{g} \\
& \leq \frac{1}{\beta} \sup _{\Sigma}|\phi|\left(1-\int_{B_{R r_{\epsilon}\left(x_{\epsilon}\right)}} \frac{1}{\lambda_{\epsilon}} u_{\epsilon}^{2} e^{\alpha_{\epsilon} u_{\epsilon}^{2}} d V_{g}\right) \\
& \leq \frac{1}{\beta} \sup _{\Sigma}|\phi|\left(1-\int_{B_{R}(0)} e^{8 \pi \varphi} d x+o_{\epsilon}(R)\right),
\end{aligned}
$$

where $o_{\epsilon}(R) \rightarrow 0$ as $\epsilon \rightarrow 0$ for any fixed $R$. Letting $\epsilon \rightarrow 0$ first, and then $R \rightarrow+\infty$, one has $I_{1} \rightarrow 0$. Then

$$
\begin{aligned}
\left|I_{2}\right| & \leq \sup _{\Sigma}|\phi| \frac{c_{\epsilon}}{\lambda_{\epsilon}} \int_{\Sigma}\left|u_{\epsilon}\right| e^{\alpha_{\epsilon} u_{\epsilon, \beta}^{2}} d V_{g} \\
& \leq \sup _{\Omega}|\phi| \frac{c_{\epsilon}}{\lambda_{\epsilon}}\left\|u_{\epsilon}\right\|_{L^{\frac{1+\beta}{1-\beta}}(\Sigma)}\left\|e^{(4 \pi-\epsilon) u_{\epsilon, \beta}^{2}}\right\|_{L^{\frac{1+\beta}{2 \beta}}(\Sigma)} \\
& \leq C \frac{c_{\epsilon}}{\lambda_{\epsilon}}
\end{aligned}
$$

for some constant $C$ depending only on $\beta$ and $\Sigma$; here we have used the Hölder inequality and the Sobolev imbedding theorem. Lemma 4.6 implies that $\lambda_{\epsilon} / c_{\epsilon} \rightarrow$ $+\infty$, whence $c_{\epsilon} / \lambda_{\epsilon} \rightarrow 0$. Hence we have $I_{2} \rightarrow 0$ as $\epsilon \rightarrow 0$. Then

$$
\begin{aligned}
I_{3} & =\int_{B_{R r_{\epsilon}}\left(x_{\epsilon}\right)} \phi \frac{c_{\epsilon}}{\lambda_{\epsilon}} u_{\epsilon} e^{\alpha_{\epsilon} u_{\epsilon}^{2}} d V_{g} \\
& =\phi\left(x_{\epsilon}+r_{\epsilon} \xi\right)\left(\int_{B_{R}(0)} e^{8 \pi \varphi} d x+o_{\epsilon}(R)\right),
\end{aligned}
$$

where $\xi \in B_{R}(0)$. As before, letting $\epsilon \rightarrow 0$ first, then $R \rightarrow+\infty$, we get $I_{3} \rightarrow \varphi(p)$. Combining all the above estimates gives (4.5). 
We need a result of Brezis and Merle [2]:

Theorem C (Brezis-Merle). Assume $\Omega \subset \mathbb{R}^{2}$ is a bounded domain and let $u \in$ $H_{0}^{1}(\Omega)$ be a weak solution of $-\triangle u=f(x)$ in $\Omega$, with $f \in L^{1}(\Omega)$. Then for any $\delta \in(0,4 \pi)$,

$$
\int_{\Omega} \exp \left[\frac{(4 \pi-\delta)|u(x)|}{\|f\|_{1}}\right] d x \leq \frac{4 \pi^{2}}{\delta}(\operatorname{diam} \Omega) .
$$

Modifying the argument of Struwe [16], we have the following:

Lemma 4.8. Assume $u \in C^{\infty}(\Sigma)$ is a solution of

$$
\left\{\begin{array}{l}
-\triangle u=f(x) \quad \text { in } \quad \Sigma, \\
\|u\|_{1} \leq c_{0}\|f\|_{1} .
\end{array}\right.
$$

Then for any $1<q<2,\|\nabla u\|_{q} \leq C\left(q, c_{0}, \Sigma\right)\|f\|_{1}$.

Proof. Without loss of generality, we assume $\|f\|_{1}=1 . \forall p \in \Sigma$, take an isothermal coordinate system $(\Omega, \phi)$ near $p$ such that $\phi(p)=0$, and $g=e^{2 h}\left(d x_{1}^{2}+d x_{2}^{2}\right)$ with $h(0)=0$. In this coordinate system, $\triangle=e^{-2 h} \triangle_{\mathbb{R}^{2}}$, and then

$$
-\triangle_{\mathbb{R}^{2}} u=e^{2 h} f \quad \text { in } \Omega .
$$

Let $v \in H_{0}^{1}(\Omega)$ be a solution of (4.7). Then we have by Theorem $\mathrm{C}$ that $e^{|v|}$ is bounded in $L^{s}(\Omega)$ for some $s>0$, whence $v$ is bounded in $L^{1}(\Omega)$. Clearly $u-v$ is harmonic in $\Omega$. By the mean value theorem, $\forall \tilde{\Omega} \Subset \Omega, \forall x \in \tilde{\Omega}$, we have for $r<\operatorname{dist}(\partial \Omega, \partial \tilde{\Omega})$

$$
\begin{aligned}
|(u-v)(x)| & =\frac{1}{\operatorname{Vol}\left(\mathbb{B}_{r}(x)\right)}\left|\int_{\mathbb{B}_{r}(x)}(u-v)(x) d x\right| \\
& \leq \frac{1}{\pi r^{2}}\left(\int_{\Sigma}|u| d V_{g}+\int_{\Omega}|v| d x\right),
\end{aligned}
$$

which implies that $u-v$ is bounded in $\tilde{\Omega}$. Therefore

$$
\int_{\tilde{\Omega}} e^{s|u(x)|} d x \leq \int_{\tilde{\Omega}} e^{s|v(x)|+s|(u-v)(x)|} d x \leq C .
$$

A covering argument implies that $e^{|u|}$ is bounded in $L^{s_{0}}(\Sigma)$ for some $s_{0}>0$.

Define $u^{+}(x)=\max \{u(x), 0\}$ and $u^{-}(x)=-\min \{u(x), 0\}$ for $x \in \Sigma$. Testing equation (4.6) by $\log \frac{1+2 u^{+}}{1+u^{+}}$, we have

$$
\int_{\Sigma} \frac{\left|\nabla u^{+}\right|^{2}}{\left(1+2 u^{+}\right)\left(1+u^{+}\right)} d V_{g} \leq \log 2 .
$$

The same inequality holds for $u^{-}$. Hence

Thus we have

$$
\int_{\Sigma} \frac{|\nabla u|^{2}}{(1+2|u|)(1+|u|)} d V_{g} \leq \log 2 .
$$

$$
\begin{aligned}
\int_{\Sigma}|\nabla u|^{q} d V_{g} & \leq \int_{\Sigma} \frac{|\nabla u|^{2}}{(1+2|u|)^{2}} d V_{g}+\int_{\Sigma}(1+2|u|)^{\frac{2 q}{2-q}} d V_{g} \\
& \leq \int_{\Sigma} \frac{|\nabla u|^{2}}{(1+2|u|)(1+|u|)} d V_{g}+\int_{\Sigma} e^{s_{0}|u|} d V_{g}+C \\
& \leq C
\end{aligned}
$$


for some constant $C$ depending only on $q, c_{0}$ and $\Sigma$. Here we use the same $C$ to denote various constants. This completes the proof of the lemma.

Lemma 4.9. $c_{\epsilon} u_{\epsilon} \rightarrow G$ weakly in $H^{1, q}(\Sigma)$ and $c_{\epsilon} u_{\epsilon} \rightarrow G$ strongly in $L^{2}(\Sigma)$ for any $1<q<2$, where $G$ is a Green function satisfying the following:

$$
\left\{\begin{array}{l}
-\Delta G=\delta_{p}+\alpha G-\frac{1}{\operatorname{Vol}(\Sigma)}, \\
\int_{\Sigma} G d V_{g}=0 .
\end{array}\right.
$$

Furthermore, $c_{\epsilon} u_{\epsilon} \rightarrow G$ in $C_{l o c}^{2}(\Sigma \backslash\{p\})$.

Proof. By (3.2), we have

$$
-\triangle\left(c_{\epsilon} u_{\epsilon}\right)=\frac{\beta_{\epsilon}}{\lambda_{\epsilon}} c_{\epsilon} u_{\epsilon} e^{\alpha_{\epsilon} u_{\epsilon}^{2}}+\gamma_{\epsilon} c_{\epsilon} u_{\epsilon}-c_{\epsilon} \frac{\mu_{\epsilon}}{\lambda_{\epsilon}} .
$$

Integration on both sides of the above equation gives $c_{\epsilon} \mu_{\epsilon} / \lambda_{\epsilon} \rightarrow 1 / \operatorname{Vol}(\Sigma)$. Let $v_{\epsilon}$ be a solution of

$$
\left\{\begin{array}{l}
-\triangle v_{\epsilon}=\frac{\beta_{\epsilon}}{\lambda_{\epsilon}} c_{\epsilon} u_{\epsilon} e^{\alpha_{\epsilon} u_{\epsilon}^{2}}-c_{\epsilon} \frac{\mu_{\epsilon}}{\lambda_{\epsilon}}-A_{\epsilon} \\
\int_{\Sigma} v_{\epsilon} d V_{g}=0
\end{array}\right.
$$

where

$$
A_{\epsilon}=\frac{1}{\operatorname{Vol}(\Sigma)} \int_{\Sigma} \frac{\beta_{\epsilon}}{\lambda_{\epsilon}} c_{\epsilon} u_{\epsilon} e^{\alpha_{\epsilon} u_{\epsilon}^{2}} d V_{g}-c_{\epsilon} \frac{\mu_{\epsilon}}{\lambda_{\epsilon}} .
$$

By Lemma 4.2 and Lemma 4.7, $A_{\epsilon}$ is bounded. Applying the Green representation formula to (4.10), we get the $L^{1}(\Sigma)$-bound of $v_{\epsilon}$. By Lemma 4.8, $v_{\epsilon}$ is bounded in $H^{1, q}(\Sigma)$ for any $1<q<2$, whence $v_{\epsilon}$ is bounded in $L^{s}(\Sigma)$ for any $s>1$. Subtracting (4.10) from (4.9), we have

$$
-\triangle\left(c_{\epsilon} u_{\epsilon}-v_{\epsilon}\right)=\gamma_{\epsilon}\left(c_{\epsilon} u_{\epsilon}-v_{\epsilon}\right)+\gamma_{\epsilon} v_{\epsilon}+A_{\epsilon} .
$$

Testing equation (4.11) by $c_{\epsilon} u_{\epsilon}-v_{\epsilon}$, we have

$$
\int_{\Sigma}\left|\nabla\left(c_{\epsilon} u_{\epsilon}-v_{\epsilon}\right)\right|^{2} d V_{g}=\gamma_{\epsilon} \int_{\Sigma}\left(c_{\epsilon} u_{\epsilon}-v_{\epsilon}\right)^{2} d V_{g}+\gamma_{\epsilon} \int_{\Sigma} v_{\epsilon}\left(c_{\epsilon} u_{\epsilon}-v_{\epsilon}\right) d V_{g} .
$$

Note that $\gamma_{\epsilon} \rightarrow \alpha<\lambda_{1}(\Sigma)$ and $\left|v_{\epsilon}\left(c_{\epsilon} u_{\epsilon}-v_{\epsilon}\right)\right| \leq \delta\left(c_{\epsilon} u_{\epsilon}-v_{\epsilon}\right)^{2}+\frac{1}{4 \delta} v_{\epsilon}^{2}$ for any $\delta>0$, one can choose $\delta<\left(\lambda_{1}(\Sigma)-\alpha\right) / 2$ and get by (4.12),

$$
\int_{\Sigma}\left|\nabla\left(c_{\epsilon} u_{\epsilon}-v_{\epsilon}\right)\right|^{2} d V_{g} \leq C \int_{\Sigma} v_{\epsilon}^{2} d V_{g}
$$

for some constant $C$ depending only on $\lambda_{1}(\Sigma)-\alpha$, provided that $\epsilon$ is sufficiently small. (4.13), together with the Poincaré inequality, gives that $c_{\epsilon} u_{\epsilon}-v_{\epsilon}$ is bounded in $H^{1,2}(\Sigma)$. Since $v_{\epsilon}$ is bounded in $H^{1, q}(\Sigma)$ for any $1<q<2$, we have $c_{\epsilon} u_{\epsilon}$ is also bounded in $H^{1, q}(\Sigma)$. Passing to a subsequence, we can assume

$$
\begin{array}{ll}
c_{\epsilon} u_{\epsilon} \rightarrow G & \text { weakly in } H^{1, q}(\Sigma), \\
c_{\epsilon} u_{\epsilon} \rightarrow G & \text { strongly in } L^{2}(\Sigma)
\end{array}
$$

for some $G \in H^{1, q}(\Sigma)$. Testing (4.9) by $\phi \in C^{\infty}(\Sigma)$, we have

$$
\begin{aligned}
\int_{\Sigma} \nabla \phi \nabla\left(c_{\epsilon} u_{\epsilon}\right) d V_{g} & =\int_{\Sigma} \phi \frac{\beta_{\epsilon}}{\lambda_{\epsilon}} c_{\epsilon} u_{\epsilon} e^{\alpha_{\epsilon} u_{\epsilon}^{2}} d V_{g}+\int_{\Sigma} \phi \gamma_{\epsilon} c_{\epsilon} u_{\epsilon} d V_{g}-c_{\epsilon} \frac{\mu_{\epsilon}}{\lambda_{\epsilon}} \int_{\Sigma} \phi d V_{g} \\
& \rightarrow \phi(p)+\alpha \int_{\Sigma} \phi G d V_{g}-\frac{1}{\operatorname{Vol}(\Sigma)} \int_{\Sigma} \phi d V_{g} .
\end{aligned}
$$

Hence (4.8) holds. 
For any fixed $\delta>0$, choose a cut-off function $\eta \in C_{0}^{\infty}\left(\Sigma \backslash B_{\delta}(p)\right)$ such that $\eta \equiv 1$ on $\Sigma \backslash B_{2 \delta}(p)$. By Lemma 4.3, we have $\left\|\nabla\left(\eta u_{\epsilon}\right)\right\|_{2} \rightarrow 0$ as $\epsilon \rightarrow 0$. Hence $e^{\eta^{2} u_{\epsilon}^{2}}$ is bounded in $L^{q}\left(\Sigma \backslash B_{\delta}(p)\right)$, whence $e^{u_{\epsilon}^{2}}$ is bounded in $L^{q}\left(\Sigma \backslash B_{2 \delta}(p)\right)$ for any $q>1$. Note that $c_{\epsilon} \mu_{\epsilon} / \lambda_{\epsilon} \rightarrow 1 / \operatorname{Vol}(\Sigma)$ as $\epsilon \rightarrow 0$, and $c_{\epsilon} u_{\epsilon}$ is bounded in $L^{r}(\Sigma)$ for any $r>2$ by the Sobolev embedding theorem. We can see from (4.9) that $-\triangle\left(c_{\epsilon} u_{\epsilon}\right)$ is bounded in $L^{q_{0}}\left(\Sigma \backslash B_{2 \delta}(p)\right)$ for some $q_{0}>2$. Applying the elliptic estimates to (4.9), we have $c_{\epsilon} u_{\epsilon} \rightarrow G$ in $C^{1}\left(\Sigma \backslash B_{3 \delta}(p)\right)$. Again by the elliptic estimates, $c_{\epsilon} u_{\epsilon} \rightarrow G$ in $C^{2}\left(\Sigma \backslash B_{4 \delta}(p)\right)$. Hence the second assertion of the lemma holds.

The proof of (1) of Theorem 1.1 follows immediately from Lemma 4.9.

\section{UPPER BOUND ESTIMATES}

In this section, we use the capacity technique to derive an upper bound of $J_{4 \pi}^{\alpha}$ under the assumption that $c_{\epsilon} \rightarrow+\infty$. The fact that capacity technique can be used here was first discovered by $\mathrm{Li}[10]$.

Let $(\Omega, \phi)$ be an isothermal coordinate system near $p$ such that $\phi(p)=0$ as in section 4 ; we still denote $\phi\left(x_{\epsilon}\right)$ by $x_{\epsilon}$ for simplicity. In such coordinates, $g=$ $e^{2 f}\left(d x_{1}^{2}+d x_{2}^{2}\right)$ with $f(0)=0$. Then we have

$$
\left|\nabla u_{\epsilon}\right|^{2} d V_{g}=\left|\nabla_{\mathbb{R}^{2}}\left(u_{\epsilon} \circ \phi^{-1}\right)\right|^{2} d x_{1} d x_{2} .
$$

Let $\mathbb{B}_{r}=\mathbb{B}_{r}\left(x_{\epsilon}\right) \subset \mathbb{R}^{2}$ be the standard ball centered at $x_{\epsilon}$ with radius $r$.

In section 4 , we have proved that $c_{\epsilon}\left(u_{\epsilon}\left(x_{\epsilon}+r_{\epsilon} x\right)-c_{\epsilon}\right) \rightarrow \varphi$ in $C_{l o c}^{2}\left(\mathbb{R}^{2}\right)$, and $c_{\epsilon} u_{\epsilon} \rightarrow G$ in $C_{l o c}^{2}(\Sigma \backslash\{p\})$. Denote $s_{\epsilon}=\sup _{\partial \mathbb{B}_{\delta}} u_{\epsilon} \circ \phi^{-1}$ and $i_{\epsilon}=\inf _{\partial \mathbb{B}_{R r_{\epsilon}}} u_{\epsilon} \circ \phi^{-1}$. Then we obtain

$$
\begin{aligned}
s_{\epsilon} & =\frac{1}{c_{\epsilon}}\left(-\frac{1}{2 \pi} \log \delta+A_{p}+o_{\delta}(1)+o_{\epsilon}(1)\right), \\
i_{\epsilon} & =c_{\epsilon}+\frac{1}{c_{\epsilon}}\left(-\frac{1}{4 \pi} \log \left(1+\pi R^{2}\right)+o_{\epsilon}(R)+o_{\epsilon}(1)\right),
\end{aligned}
$$

where $o_{\epsilon}(1) \rightarrow 0$ as $\epsilon \rightarrow 0, o_{\delta}(1) \rightarrow 0$ as $\delta \rightarrow 0$, and $o_{\epsilon}(R) \rightarrow 0$ for any fixed $R>0$ as $\epsilon \rightarrow 0$. Define a function space

$$
\mathcal{T}_{\epsilon}=\left\{u \in H^{1,2}\left(\mathbb{B}_{\delta} \backslash \mathbb{B}_{R r_{\epsilon}}\right):\left.u\right|_{\partial \mathbb{B}_{\delta}}=s_{\epsilon},\left.u\right|_{\partial \mathbb{B}_{R r_{\epsilon}}}=i_{\epsilon}\right\} .
$$

It is not difficult to see that

$$
\inf _{u \in \mathcal{T}_{\epsilon}} \int_{\mathbb{B}_{\delta} \backslash \mathbb{B}_{R r_{\epsilon}}}\left|\nabla_{\mathbb{R}^{2}} u\right|^{2} d x_{1} d x_{2}
$$

is attained by $w(x)$ satisfying

$$
\left\{\begin{array}{l}
\triangle_{\mathbb{R}^{2}} w=0 \quad \text { in } \quad \mathbb{B}_{\delta} \backslash \overline{\mathbb{B}}_{R r_{\epsilon}}, \\
\left.w\right|_{\partial \mathbb{B}_{\delta}}=s_{\epsilon}, \\
\left.w\right|_{\partial \mathbb{B}_{R r_{\epsilon}}}=i_{\epsilon} .
\end{array}\right.
$$

One can check that

$$
w(x)=\frac{s_{\epsilon}\left(\log \left|x-x_{\epsilon}\right|-\log \left(R r_{\epsilon}\right)\right)+i_{\epsilon}\left(\log \delta-\log \left|x-x_{\epsilon}\right|\right)}{\log \delta-\log \left(R r_{\epsilon}\right)},
$$

whence

$$
\int_{\mathbb{B}_{\delta} \backslash \mathbb{B}_{R r_{\epsilon}}}\left|\nabla_{\mathbb{R}^{2}} w\right|^{2} d x_{1} d x_{2}=\frac{2 \pi\left(s_{\epsilon}-i_{\epsilon}\right)^{2}}{\log \delta-\log \left(R r_{\epsilon}\right)} .
$$


Let $\tilde{u}_{\epsilon}=\max \left\{s_{\epsilon}, \min \left\{u_{\epsilon}, i_{\epsilon}\right\}\right\}$. Then $\tilde{u}_{\epsilon} \circ \phi^{-1} \in \mathcal{T}_{\epsilon}$, whence

$$
\begin{aligned}
& \int_{\mathbb{B}_{\delta} \backslash \mathbb{B}_{R r_{\epsilon}}}\left|\nabla_{\mathbb{R}^{2}} w\right|^{2} d x_{1} d x_{2} \\
& \leq \int_{\mathbb{B}_{\delta} \backslash \mathbb{B}_{R r_{\epsilon}}}\left|\nabla_{\mathbb{R}^{2}}\left(\tilde{u}_{\epsilon} \circ \phi^{-1}\right)\right|^{2} d x_{1} d x_{2} \\
& =\int_{\phi^{-1}\left(\mathbb{B}_{\delta}\right) \backslash \phi^{-1}\left(\mathbb{B}_{R r_{\epsilon}}\right)}\left|\nabla \tilde{u}_{\epsilon}\right|^{2} d V_{g}\left|\nabla u_{\epsilon}\right|^{2} d V_{g}-\int_{\phi^{-1}\left(\mathbb{B}_{\left.R r_{\epsilon}\right)}\right)}\left|\nabla u_{\epsilon}\right|^{2} d V_{g} . \\
& \leq 1-\int_{\Sigma \backslash \phi^{-1}\left(\mathbb{B}_{\delta}\right)}
\end{aligned}
$$

Now we compute $\int_{\phi^{-1}\left(\mathbb{B}_{R r_{\epsilon}}\right)}\left|\nabla u_{\epsilon}\right|^{2} d V_{g}$ and $\int_{\Sigma \backslash \phi^{-1}\left(\mathbb{B}_{\delta}\right)}\left|\nabla u_{\epsilon}\right|^{2} d V_{g}$. Recall that $G=$ $-\frac{\log r}{2 \pi}+\beta$, where $\beta=A_{p}+O(r)$ and $\beta \in C^{1}(\Sigma)$. Using equation (4.8) and the divergence theorem, we have

$$
\int_{\Sigma \backslash \phi^{-1}\left(\mathbb{B}_{\delta}\right)}|\nabla G|^{2} d V_{g}=-\frac{1}{2 \pi} \log \delta+A_{p}+\alpha\|G\|_{2}^{2}+o_{\epsilon}(\delta)+o_{\epsilon}(1)+o_{\delta}(1),
$$

where $o_{\epsilon}(\delta) \rightarrow 0$ for any fixed $\delta>0$ as $\epsilon \rightarrow 0$. By Lemma 4.9, $c_{\epsilon} u_{\epsilon} \rightarrow G$ in $C_{l o c}^{2}(\Omega \backslash\{p\})$, and we obtain

$$
\int_{\Sigma \backslash \phi^{-1}\left(\mathbb{B}_{\delta}\right)}\left|\nabla u_{\epsilon}\right|^{2} d V_{g}=\frac{1}{c_{\epsilon}^{2}}\left(-\frac{\log \delta}{2 \pi}+A_{p}+\alpha\|G\|_{2}^{2}+o_{\epsilon}(\delta)+o_{\epsilon}(1)+o_{\delta}(1)\right) .
$$

On the other hand,

$$
\int_{B_{R}(0)}|\nabla \varphi|^{2} d x=\frac{1}{4 \pi} \log \left(1+\pi R^{2}\right)-\frac{1}{4 \pi}+o_{R}(1),
$$

where $o_{R}(1) \rightarrow 0$ as $R \rightarrow+\infty$. Hence by the fact that

$$
c_{\epsilon}\left(u_{\epsilon}\left(x_{\epsilon}+r_{\epsilon} x\right)-c_{\epsilon}\right) \rightarrow \varphi(x) \text { in } C_{l o c}^{2}\left(\mathbb{R}^{2}\right),
$$

we have

$$
\int_{\phi^{-1}\left(\mathbb{B}_{R r_{\epsilon}}\right)}\left|\nabla u_{\epsilon}\right|^{2} d V_{g}=\frac{1}{c_{\epsilon}^{2}}\left(\frac{1}{4 \pi} \log \left(1+\pi R^{2}\right)-\frac{1}{4 \pi}+o_{R}(1)+o_{\epsilon}(R)+o_{\epsilon}(1)\right) .
$$

Recalling $r_{\epsilon}^{2}=\lambda_{\epsilon} /\left(\beta_{\epsilon} c_{\epsilon}^{2}\right) e^{-\alpha_{\epsilon} c_{\epsilon}^{2}}$, one gets

$$
\frac{1}{2 \pi}\left(\log (\delta)-\log \left(R r_{\epsilon}\right)\right)=\frac{\log \delta-\log R}{2 \pi}-\frac{1}{4 \pi} \log \frac{1}{\beta_{\epsilon}}-\frac{1}{4 \pi} \log \frac{\lambda_{\epsilon}}{c_{\epsilon}^{2}}+\frac{\alpha_{\epsilon} c_{\epsilon}^{2}}{4 \pi} .
$$

From (5.1) to (5.5), we obtain

$$
\begin{aligned}
& c_{\epsilon}^{2}+2\left(-\frac{1}{4 \pi} \log \left(1+\pi R^{2}\right)+\frac{1}{2 \pi} \log \delta-A_{p}\right)+o_{\epsilon}(\delta)+o_{\epsilon}(R)+o_{\epsilon}(1) \\
& \leq\left(\frac{\log \delta-\log R}{2 \pi}-\frac{1}{4 \pi} \log \frac{\lambda_{\epsilon}}{c_{\epsilon}^{2}}+\frac{\alpha_{\epsilon}}{4 \pi} c_{\epsilon}^{2}+o_{\epsilon}(1)\right) \times(1- \\
& \left.\frac{\frac{1}{4 \pi} \log \left(1+\pi R^{2}\right)-\frac{1}{4 \pi}-\frac{1}{2 \pi} \log \delta+A_{p}+\alpha\|G\|_{2}^{2}+o_{\epsilon}(\delta)+o_{\epsilon}(R)+o_{\delta}(1)+o_{R}(1)}{c_{\epsilon}^{2}}\right),
\end{aligned}
$$


which implies that

$$
\begin{aligned}
\log \frac{\lambda_{\epsilon}}{c_{\epsilon}^{2}} \quad \leq 4 \pi \alpha c_{\epsilon}^{2}\left\|u_{\epsilon}\right\|_{2}^{2}+4 \pi A_{p}+1+\log \pi-4 \pi \alpha\|G\|_{2}^{2} \\
+o_{\epsilon}(\delta)+o_{\epsilon}(R)+o_{\epsilon}(1)+o_{\delta}(1)+o_{R}(1)
\end{aligned}
$$

By Lemma 4.9,

$$
\lim _{\epsilon \rightarrow 0} c_{\epsilon}^{2}\left\|u_{\epsilon}\right\|_{2}^{2}=\|G\|_{2}^{2} .
$$

Letting $\epsilon \rightarrow 0$ first, then $R \rightarrow+\infty$ and $\delta \rightarrow 0$, we obtain by (5.6),

$$
\limsup _{\epsilon \rightarrow 0} \frac{\lambda_{\epsilon}}{c_{\epsilon}^{2}} \leq \pi e^{1+4 \pi A_{p}}
$$

whence by Lemma 4.6,

$$
\sup _{u \in \mathcal{H}} J_{4 \pi}^{\alpha}(u)=\limsup _{\epsilon \rightarrow 0} \int_{\Sigma} e^{\alpha_{\epsilon} u_{\epsilon}^{2}} d V_{g} \leq \operatorname{Vol}(\Sigma)+\pi e^{1+4 \pi A_{p}} .
$$

In fact we have proved the following:

Proposition 5.1. Under the assumption that $c_{\epsilon} \rightarrow+\infty$, it follows that

$$
\sup _{u \in \mathcal{H}} J_{4 \pi}^{\alpha}(u) \leq \operatorname{Vol}(\Sigma)+\pi e^{1+4 \pi A_{p}} .
$$

\section{The EXIstence Result}

In this section, we will construct a blow-up sequence $\phi_{\epsilon}$ such that $\left\|\nabla \phi_{\epsilon}\right\|_{2}=1$ and

$$
\int_{\Sigma} e^{4 \pi\left(\phi_{\epsilon}-\bar{\phi}_{\epsilon}\right)^{2}\left(1+\alpha\left\|\phi_{\epsilon}-\bar{\phi}_{\epsilon}\right\|_{2}^{2}\right)} d V_{g}>\operatorname{Vol}(\Sigma)+\pi e^{1+4 \pi A_{p}}
$$

for sufficiently small $\alpha, \epsilon>0$, where $\bar{\phi}_{\epsilon}=\frac{1}{V o l(\Sigma)} \int_{\Sigma} \phi_{\epsilon} d V_{g}$. The contradiction between (6.1) and Proposition 5.1 implies that $c_{\epsilon}$ is bounded. Applying elliptic estimates to equation (3.2), we have $u_{\epsilon} \rightarrow u_{\alpha}$ in $C^{\infty}(\Sigma)$ for some $u_{\alpha} \in C^{\infty}(\Sigma) \cap \mathcal{H}$. Hence the point (3) of Theorem 1.1 holds.

To prove $(6.1)$, we set $\tilde{\beta}=G+\frac{1}{2 \pi} \log r-A_{p}$; hence $\tilde{\beta}=O(r)$. Here $r(x)=$ $\operatorname{dist}(x, p)$. Set

$$
\phi_{\epsilon}= \begin{cases}\frac{c+\frac{1}{c}\left(-\frac{1}{4 \pi} \log \left(1+\pi \frac{r^{2}}{\epsilon^{2}}\right)+B\right)}{\sqrt{1+\frac{\alpha}{c^{2}}\|G\|_{2}^{2}}} & \text { for } \quad r \leq R \epsilon, \\ \frac{1}{\sqrt{c^{2}+\alpha\|G\|_{2}^{2}}}(G-\eta \tilde{\beta}) & \text { for } \quad R \epsilon<r<2 R \epsilon, \\ \frac{1}{\sqrt{c^{2}+\alpha\|G\|_{2}^{2}}} G & \text { for } \quad r \geq 2 R \epsilon,\end{cases}
$$

where $\eta \in C_{0}^{\infty}\left(B_{2 R \epsilon}(p)\right)$ is a cutoff function, $\eta=1$ on $B_{R \epsilon}(p),\|\nabla \eta\|_{L^{\infty}}=O\left(\frac{1}{R \epsilon}\right)$, $B$ is a constant to be determined later, and $R, c$ depending on $\epsilon$ will also be chosen later such that $R \epsilon \rightarrow 0$ and $R \rightarrow+\infty$. In order to ensure that $\phi_{\epsilon} \in H^{1,2}(\Sigma)$, we set

$$
c+\frac{1}{c}\left(-\frac{1}{4 \pi} \log \left(1+\pi R^{2}\right)+B\right)=\frac{1}{c}\left(-\frac{1}{2 \pi} \log (R \epsilon)+A_{p}\right),
$$

which gives

$$
2 \pi c^{2}=-2 \log \epsilon-2 \pi B+2 \pi A_{p}+\frac{1}{2} \log \pi+O\left(\frac{1}{R^{2}}\right) .
$$


By a direct calculation,

$$
\begin{aligned}
\int_{\Sigma}\left|\nabla \phi_{\epsilon}\right|^{2} d V_{g}= & \frac{1}{c^{2}+\alpha\|G\|_{2}^{2}}\left(-\frac{\log \epsilon}{2 \pi}+\frac{\log \pi}{4 \pi}-\frac{1}{4 \pi}+A_{p}+\alpha\|G\|_{2}^{2}\right. \\
& \left.+O\left(\frac{1}{R^{2}}\right)+O(R \epsilon \log (R \epsilon))\right) .
\end{aligned}
$$

To ensure $\left\|\nabla \phi_{\epsilon}\right\|_{2}=1$, we set

$$
c^{2}=-\frac{\log \epsilon}{2 \pi}+\frac{\log \pi}{4 \pi}-\frac{1}{4 \pi}+A_{p}+O\left(\frac{1}{R^{2}}\right)+O(R \epsilon \log (R \epsilon)) .
$$

By (6.2) and (6.3), we have

$$
B=\frac{1}{4 \pi}+O\left(\frac{1}{R^{2}}\right)+O(R \epsilon \log (R \epsilon)) .
$$

Setting $R=-\log \epsilon$, one gets $\bar{\phi}_{\epsilon}=O\left((R \epsilon)^{2} \log \epsilon\right)$. A straightforward calculation shows

$$
\int_{B_{R \epsilon}(p)} e^{4 \pi\left(\phi_{\epsilon}-\bar{\phi}_{\epsilon}\right)^{2}\left(1+\alpha\left\|\phi_{\epsilon}-\bar{\phi}_{\epsilon}\right\|_{2}^{2}\right)} d V_{g} \geq \pi e^{1+4 \pi A_{p}}+\alpha^{2}\|G\|_{2}^{4} O\left(\frac{1}{\log \epsilon}\right)+O\left(\frac{\log \log \epsilon}{(\log \epsilon)^{2}}\right) .
$$

On the other hand,

$$
\begin{aligned}
\int_{\Sigma \backslash B_{R \epsilon}(p)} e^{4 \pi\left(\phi_{\epsilon}-\bar{\phi}_{\epsilon}\right)^{2}\left(1+\alpha\left\|\phi_{\epsilon}-\bar{\phi}_{\epsilon}\right\|_{2}^{2}\right)} d V_{g} & \geq \int_{\Sigma \backslash B_{2 R \epsilon}(p)}\left(1+4 \pi\left(\phi_{\epsilon}-\bar{\phi}_{\epsilon}\right)^{2}\right) d V_{g} \\
& \geq \operatorname{Vol}(\Sigma)+\frac{8 \pi^{2}}{\log \frac{1}{\epsilon}} \int_{\Sigma} G^{2} d x+o\left(\frac{1}{\log \epsilon}\right) .
\end{aligned}
$$

Hence, we have

$$
J_{4 \pi}^{\alpha}\left(\phi_{\epsilon}-\bar{\phi}_{\epsilon}\right)>\operatorname{Vol}(\Sigma)+\pi e^{1+4 \pi A_{p}}
$$

for sufficiently small $\alpha, \epsilon>0$, and (6.1) holds.

\section{ACKNOWLEDGEMENTS}

The author is indebted to Yuxiang Li for many helpful suggestions. He also thanks the referee for valuable comments on the manuscript. This work was partly supported by the NSFC 10601065.

\section{REFERENCES}

[1] Adimurthi and O. Druet: Blow-up analysis in dimension 2 and a sharp form of TrudingerMoser inequality. Comm. in PDE., 29(1): 295-322, 2004. MR2038154 (2005a:46064)

[2] H. Brezis and F. Merle: Uniform Estimates and Blow-up Behavior for Solutions of $-\Delta u=$ $V(x) e^{u}$ in Two Dimensions, Commun In PDE 16, 1223-1253 (1991). MR1132783 (92m:35084)

[3] L. Carleson and A. Chang: On the existence of an extremal function for an inequality of J. Moser. Bull. Sci. Math., 110: 113-127, 1986. MR0878016 (88f:46070)

[4] W. Chen and C. Li: Classification of solutions of some nonlinear elliptic equations. Duke Math. J. 63: 615-622, 1991. MR1121147 (93e:35009)

[5] W. Y. Ding, J. Jost, J. Li and G. Wang: The differential equation $-\Delta u=8 \pi-8 \pi h e^{u}$ on a compact Riemann Surface. Asian J. Math., 1(2): 230-248, 1997. MR1491984 (98k:58062)

[6] O. Druet and E. Hebey: The AB program in geometric analysis. Sharp Sobolev inequalities and related problems. Memoirs of the AMS, 106: 761, 2002. MR1938183 (2003m:58028)

[7] J. Escobar and R. Schoen: Conformal metrics with prescribed scalar curvature. Inventiones Math., 86: 243-253, 1986. MR0856845 (88b:58030)

[8] M. Flucher: Extremal functions for the Trudinger-Moser inequality in 2 dimensions. Comment. Math. Helv., 67: 471-497, 1992. MR1171306 (93k:58073) 
[9] L. Fontana: Sharp borderline Sobolev inequalities on compact Riemannian manifolds. Comm. Math. Helv., 68: 415- 454, 1993. MR1236762 (94h:46048)

[10] Y. Li: Moser-Trudinger inequality on compact Riemannian manifolds of dimension two. J. Partial Differential Equations 14, no. 2, 163-192, 2001. MR1838044 (2002h:58033)

[11] Y. Li and P. Liu: A Moser-Trudinger inequality on the boundary of a compact Riemann surface. Math. Z., 250: 363-386, 2005. MR2178789

[12] K.C. Lin: Extremal functions for Moser's inequality. Trans. Amer. Math. Sco., 348:2663-2671, 1996. MR1333394 (96i:58043)

[13] P. L. Lions: The concentration-compactness principle in the calculus of variations, the limit case, part I. Rev. Mat. Iberoamericana, 1: 145-201, 1985. MR0834360 (87c:49007)

[14] J. Moser: A sharp form of an Inequality by N. Trudinger. Ind. Univ. Math. J., 20:1077-1091, 1971. MR0301504 (46:662)

[15] R. Schoen: Conformal deformation of a Riemannian metric to a constant scalar curvature. J. Differential Geometry, 20: 479-495, 1984. MR0788292 (86i:58137)

[16] M. Struwe: Positive solutions of critical semilinear elliptic equations on non-contractible planar domains. J. Eur. Math. Soc., 2(4):329-388, 2000. MR1796963 (2001h:35070)

[17] N. S. Trudinger: On embeddings into Orlicz spaces and some applications. J. Math. Mech., 17: 473-484, 1967. MR0216286 (35:7121)

Department of Mathematics, Information School, Renmin University of China, BeiJing 100872, PeOple's Republic of ChinA

E-mail address: yunyan_yang2002@yahoo.com.cn 